Proceedings of the 2002 IEEE/RSJ

Intl. Conference on Intelligent Robots and Systems

EPFL, Lausanne, Switzerland • October 2002

\title{
A Quantitative Technique for Analysing Robot-Human Interactions
}

\author{
Kerstin Dautenhahn* \\ Iain Werry** \\ *Adaptive Systems Research Group, University of Hertfordshire, Hatfield, UK, K.Dautenhahn@herts.ac.uk \\ ${ }^{* *}$ Department of Cybernetics, University of Reading, UK, Iain@aurora-project.com
}

\begin{abstract}
This paper proposes a technique for quantitatively describing and analysing robot-human interactions in terms of low-level behavioural criteria (so-called micro-behaviours). In order to demonstrate the usefulness of this technique, we describe a case study that was conducted as part of the Aurora project where we develop robotic toys as therapeutic tools for children with autism. In this project we made explicit choices of how to assess robot-human interaction and how to study the impact of interaction. Results of a comparative study with autistic children are shown where we focus on eye gaze behaviour. The results point out common tendencies as well as clear differences among the children, important information for future development of robots in the Aurora project. We propose that this technique is applicable to a wide range of application areas that involve robot-human interactions. The work presented in this paper is intended to open up a discussion on appropriate techniques to systematically assess robot-human interactions. Such research is important for the development of robots in human-inhabited environments.
\end{abstract}

\section{Introduction}

With the development of more and more complex robots to be used in rehabilitation, entertainment, service or other applications with the 'human in the loop', robot-human interaction is a rapidly growing area of research. This paper results from research carried out in the Aurora project which studies the usage of interactive robots in autism therapy (section 2). An important question in this project is how to analyse robot-human interactions. In this paper we describe an analysis technique that we developed, inspired by an existing technique used in psychology that is based on 'micro-behaviours'. We show its application in a comparative case study (section 3). The paper concludes by discussing advantages and disadvantages of the approach and the potential applicability of the technique to other application areas (section 4).

\section{Application Area}

\subsection{The Therapeutic Context: Autism}

Despite huge differences in abilities of people diagnosed along the autistic spectrum, they generally have in common particular deficits in social interaction, communication, imagination and fantasy [9]. On the one hand, to an autistic person other people's social behaviour often appears overwhelming, unpredictable, confusing and therefore threatening. On the other hand, many people with autism enjoy using computers or other machines that are predictable and provide a 'safe' environment for them, away from the demands of a social environment that they cannot make sense of and that they cannot 'properly' respond to. Also, people with autism usually focus on the 'literal meaning' and details of things. Computers and other machines are much closer to this perspective of 'literal meaning' since they follow simple rules in a predictable way which requires less 'interpretation' than e.g. a human's facial expressions or subtleties of human language.

\subsection{Interactive Systems in Autism Therapy}

For over thirty years researchers have been investigating how to use robots in education [13], [8]. The use of computers in autism therapy is well studied ([3], [15], [12]). Increasingly researchers study the application of software and robotic systems in autism therapy. Seminal work done in the early 1970ies in Edinburgh with one autistic child controlling a teleoperated (non-interactive) robot gave first encouraging ideas [18] about a potential therapeutic role of robots. More recently interactive robotic systems are being studied [4], [11], [14].

Note, that the use of interactive systems in autism therapy is a very recent development. Research in that area has widely exploratory character. Due to the relative lack of previous work that one can build on, a lot of experimental and methodological 'ground work' needs to be done, such as the development of appropriate analysis and evaluation techniques. Also, due to the specific nature of the application area sample sizes are usually small and heteroge- 
neous with regard to the interaction competencies of the subjects.

\subsection{The Aurora Project}

The work discussed in this paper is part of the longterm project Aurora [1] which studies systematically since 1998 how robots with simple interaction skills can be used as a teaching device ('toys') in autism therapy. Aurora stands for "Autonomous robotic platform as a remedial tool for children with autism". Our work focuses on autism therapy, rather than studying the precise nature of autism. We are therefore mainly interested in analysis techniques that are little theoretically biased.

Robot-human interactions in the Aurora project are widely unconstrained and unstructured, children are encouraged to explore and 'discover' their interaction skills rather than being taught explicitly. In the trials reported here we used an autonomous mobile robot which shows a few basic behaviours of approach and avoidance. In these trials, the children are allowed to interact with the robot in whatever position they prefer (e.g. lying on the floor, crawling, standing), they are also free to choose how they interact with the robot (touching, approaching, watching from a distance, picking it up etc.). In the general context of robot-human interactions these conditions are much different from other projects on robot-human interaction (e.g. work on Kismet [2]) where the human is expected to interact with the robot while adopting a particular position and orientation towards the robot (e.g. sitting face-to-face in close distance to an interactive robot that is not moving in space, or standing in front of a mobile robot). The mobile robot that we are using in the Aurora project allows unconstrained, full-body interactions. The autistic children who are interacting with the robot are between 8-12 years of age. For more background on the project's robotic and therapeutic issues, as well as results see e.g. ([6], [19]; [20], [7]). Generally, our observations of interactions between children with autism and robots to date indicate that the robot can engage the children with autism successfully in playful interactions.

\section{A Comparative Study}

The comparative study that this paper focuses on was conducted at Radlett Lodge School in Hertfordshire, England, a school of the National Autistic Society (NAS).

\subsection{Hypothesis}

Our basic starting point in the Aurora project is that we can develop a robot as a useful therapeutic tool. We assume that playing with an interactive robot can be beneficial to the children, as opposed to interactions with other already available toys. The rationale of this argument is explained in more detail e.g. in [4] and [6].

However, if we want to argue that interactions with the robot are 'special' then we need to establish that children interact differently with robots than with other toys, and we need to be able to describe these differences in detail. In our comparative study we expose each child in two different time intervals first to a non-interactive object that looks similar to the robot, then to the interactive robot. We compare robot-human interactions in both conditions.

\subsection{Set up}

A familiar teacher is always present in the room where the trials are conducted, in addition to one or two experimenters. The experimenters do not initiate communication or interaction with the child, but they respond when addressed by the child. The teacher is generally only observing, occasionally interfering when the child wants to leave the room, is about to destroy the robot, etc.

The robotic platform used in this research is a Labo-1 type robot, provided by Applied AI Inc. The platform is $30 \mathrm{~cm}$ wide by $40 \mathrm{~cm}$ long and weighs $6.5 \mathrm{~kg}$. It is equipped with eight infrared sensors (four at the front, two at the rear and one at either side), as well as a heat sensor on a swivel mount at the front of the robot. Using these sensors, the robot is able to avoid obstacles and can follow a heat source such as a child. Additionally, the robot has been upgraded to include a speech synthesiser unit and is able to produce short spoken phrases using a neutral intonation. The robot is heavy enough to be difficult for the children to pick up and is robust enough to survive an average trial, including being pushed around and stepped on.

The programming of the robot allows it to perform basic actions, such as avoiding obstacles, following children and producing speech. This creates a robot that will try to approach the child, respond vocally to his presence, and avoid other obstacles - as well as not coming into actual contact with the child. The robot is able to play simple interaction games with the children, such as the 'chasing game' and the 'following game'. A key element in both games is turn-taking, a central element that regulates humanhuman interaction and that is a therapeutically important behaviour. The robot is programmed so that during the following game it follows the child around in the room. The child (e.g. when lying on the floor) can move his legs/arms so that the robot approaches, or the child walks away and waits for the robot to follow. The children often walk around the robot or 
step aside in order to 'test' if the robot can see them and check if it follows. In the chasing game the child might discover that when he approaches the robot it moves backwards, eventually child and robot take turns in approach/avoidance games. If the child does not react for a while then the robot tries to engage the child in interaction by using programmed movements and synthetic speech such as the phrase "Can't see you". Generally, the children very much enjoy the interactions, we observe many examples of positive expressions such as smiling, laughing etc. during interactions with the mobile robot.

All trials are videotaped for later analysis.

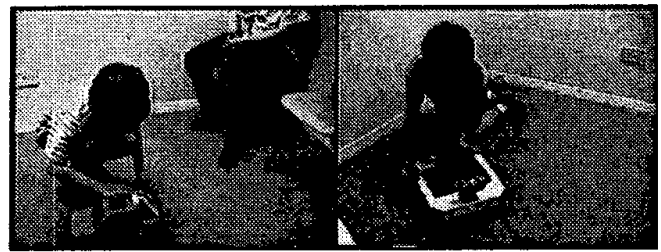

Figure 1: Ivan playing with the toy truck (left) and the robot (right). All names of children used in this paper are pseudonyms.

\subsection{Analysing Robot-Human Interactions}

How can robot-human interaction be analysed? Descriptive narratives describing how autistic children interact with robots, as used e.g. in ([6], [11]), are useful in order to give first impressions of what kind of interactions occur. However, in order to make progress towards learning about how to use robots in autism therapy a more systematic approach is needed. Since many of our children are non-verbal, questionnaires or interviews are not suitable in order to assess the robot's 'success' in terms of engaging the children in interactions. Many techniques usually used in human-computer interface design are not appropriate in our application domain. Generally, we believe that in many application domains involving robots and humans observational data is highly valuable, in order to avoid e.g. the social desirability response bias (where people respond to studies in a way that presents them in a positive light), or the interviewer bias (where the interviewer influences the responses) ${ }^{1}$.

In the robotics community robot-human interaction is often assessed either by focusing on the impact on the user (employing questionnaires, interviews etc.), e.g. for studying how well people recognise a robot's emotional expressions, or assessing the performance of the robot, namely how good the robot has achieved

\footnotetext{
${ }^{1}$ For a discussion of advantages and disadvantages of different methods see e.g. [16].
}

a certain goal such as navigating an area or delivering items. Such techniques cannot be applied in the Aurora project since here the robot does not have an explicit 'task', it is not required to solve any 'problems' (from the robot's point of view) apart from interacting with the children and, a more indirect and abstract goal, structuring these interactions so that interactions address therapeutically relevant issues.

The method we describe in this paper is strongly influenced by Tardiff et al. [17] who analysed interactions in child-adult dyads. The group studied 5-14 year old autistic children who were interacting with an adult and playing with/without objects. Later different categories were applied in the detailed analysis of videotapes of the child's and the adult's behaviour, including behaviours such as gaze, pointing, touch, operate etc. Once these micro-behaviours are coded on a second-by-second basis, statistical techniques can be applied. Note, that these categories refer to micro-behaviours which has several advantages:

(a) The micro-behaviours are well identifiable, they are rather low-level and 'action/movement'- oriented categories

(b) The micro-behaviours are not very specific to autism, they can thus be identified more easily by researchers or anybody who does not have intensive contact with autistic children on a daily basis, and who does not possess expert knowledge on the nature of autism

(c) Ultimately, micro-behaviours are more likely to be recognisable by a computational vision system. An automatic recognition and analysis system that receives video input on robot-child interactions and can output an analysis of interactional patterns would be fundamentally helpful for researchers developing therapeutic robotic toys for children, assisting in the time-consuming handcoding of the video data. Given the rapid progress in technology necessary to recognise facial expressions, gestures, movements etc. this goal seems not too unrealistic.

\subsection{Method Used}

The analysis of the videotapes focuses on the child, not on the robot, teachers or experimenters present. However, since we are trying to promote social interaction and communication, the presence of other people is not ignored, rather analysed from the perspective of the child.

Trials are conducted in three sections. The first section involves the child interacting with a (commercially available) toy truck that has approximately the same size as the robotic platform (cf. fig. 1, left). We selected the toy truck because of its suitable size, and 
because we needed a comparative non-interactive object that is very different from the robot. The second section consists of both the toy truck and the robot present, although the robot is switched off and thus passive. The third section uses the robot in an 'active mode' (cf. fig. 1, right). Note, that in half the trials the order is reversed, i.e. the robot is presented in the first section and the toy truck in the third. This structure allows us to compare interactions with the robot with those of a solely passive object. Timing of the sections may vary, typically the first and third section are four minutes while the second section is two minutes, however this is altered by observations from the teachers and experimenters present depending on the enjoyment of the child. In initial tests the 4-2-4 minute interval lengths were shown to be appropriate.

The trial video is segmented into one-second intervals, and each second is analysed for the presence of various behaviours and actions by the child after Tardiff et al. (1995), with categories altered for our particular application involving robots. The manual coding and subsequent analysis of the video data is done by one of the experimenters. Since the sections of the trials are not always of the same length, averages of total section times are taken.

Trials are analysed using a set of fourteen criteria, which are broken into two general categories. The first category consists of the criteria eye gaze, eye contact, operate, handling, touch, approach, move away and attention. This category depends on a focus of the action or behaviour and this focus further categorises the analysis of the behaviour. The second category consists of the criteria vocalisation, speech, verbal stereotype, repetition and blank. While the focus of these actions are recorded where possible, it is not always obvious what the focus is, or even if there is one at all. For example, a child may utter a short sound as a result of an action of the robot, the teacher, an internal stimulus (such as hunger), the sun appearing from behind a cloud or for no obvious reason aside from the desire to do so. Additionally, the criterion 'repeat' is used for actions which are repeated by the child without a repeated stimulus, and the criterion of 'blank' is recorded for cases when the child does not appear to perform any observable action. An extra criterion of 'other' allows us to capture any behaviours and actions not considered prior to the trials and which do not fall into one of the above categories, and to make notes on the nature of this behaviour. The fourteen criteria can be described as follows:

Eye Gaze: Direction of gaze

Eye Contact: Child making eye contact with a person, gazing at the front of the toy truck, or the heat sensor of the robot which has an 'eye-like' appearance

Operate: Manipulating an object to make it work, e.g. an IR sensor in order to control the robot

Handling: e.g. picking up, pushing, etc - includes an element of inquisitiveness, including pressing buttons

Touch: Physical contact - child initiated

Approach: Moving towards. Must be a deliberate movement

Move Away: Must be a deliberate movement away from object

Vocalisation: Sounds such as yells, mumbling, including whistling (start of vocalisation)

Speech: Word utterances, not necessarily coherent, but a string of words (start of speech)

Verbal Stereo: Echolalia, non-speech sounds with repetition or without clear purpose

Repetition: Any behaviour or action which can be grouped and sections repeated, specifically 'autistic behaviours' such as spinning wheels or other distinct repetitive behaviours that autistic children often show

Attention: The apparent focus of the child's attention, e.g. robot or toy truck

Other: Actions that are as yet unclassified, or notes (for example reactions/interactions to people, distress/boredom of child, symbolic play such as stories/play, etc.)

Blank: No or very little visible child action or introspective behaviour without external purpose, e.g. sitting almost motionless

A well known issue in social sciences, ethology, psychology and other fields that use analysis methods with manual coding schemes is interrater reliability, namely the extent to which another observer achieves the same results. This is necessary for showing that the method and the results achieved are not substantially biased, i.e. influenced by subjective judgements. We compared the results for truck and robot for eye gaze and one child, using a 'naive' second observer who was not involved in the trials, and who was not aware of the results of the first observer. Results show no statistically significant differences between the behaviour lengths rated by the two observers (Kolmogorov-Smirnow test). This indicates a good interrater reliability of the technique.

\subsection{Results}

Behaviour length data from three (Adam, Ivan, Sean) out of the six children that could be statistically tested (excluding Oscar) showed significant differences in gaze duration between the conditions (truck, robot), although not in the same di- 


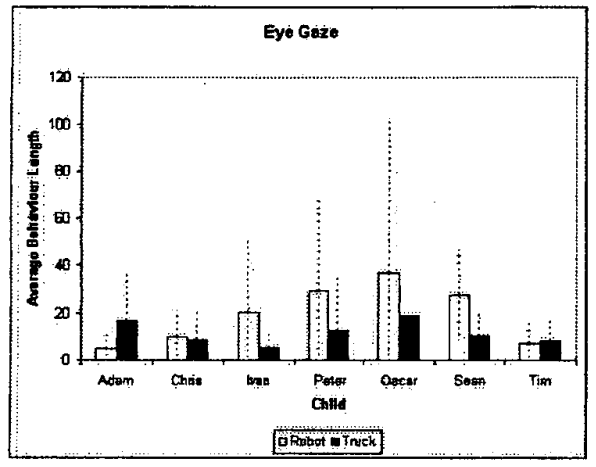

Figure 2: Histogram showing comparative eye gaze behaviours of seven children directed towards robot and truck. Averages and standard deviations (dotted lines) are shown.

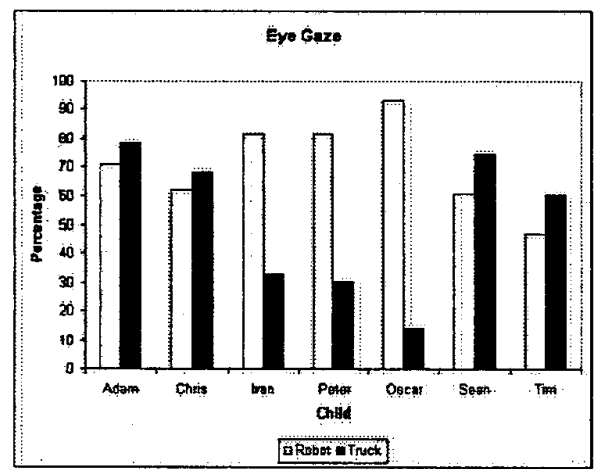

Figure 3: Histogram showing comparative eye gaze behaviours in terms of percentages (total duration of eye gaze behaviour in relation to the duration of the section).



Figure 4: Scatter plot with average behaviour lengths robot/truck. Groups can be identified: group one (circle), two (square) and three (triangle). Averages and standard deviations (dotted lines) are shown. rection ${ }^{2}$. Combining the probabilities showed statistically significant differences between the two conditions (truck, robot) ${ }^{3}$.

The following figures are based on mean values. Figure 2 compares eye gaze behaviour directed towards the robot/truck for the seven children studied.

Figure 3 compares the percentages of eye gaze behaviour shown in sections one and three with respect to the section lengths.

Figure 4 shows a xy-plot that depicts average behaviour lengths directed to the robot in relation to average behaviour lengths directed towards the truck.

\subsection{Discussion}

In figure 2 the histogram shows clear differences in mean values for eye gaze behaviour. Three groups can be identified: First, 4 of 7 children, namely Ivan, Peter, Oscar and Sean, show clearly more eye gaze behaviour towards the robot than towards the toy truck (group one). For the second group with Chris and Tim the result is more balanced. Lastly, Adam shows significantly more eye gaze behaviour directed towards the toy truck than to the robot. A further analysis of the videos with respect to the attention criterium explains Adam's somewhat unusual behaviour (as compared to the other children in the group). The trial lengths for Adam are 8 minutes and 12 seconds for section one (interaction with the robot) and 6 minutes and 2 seconds for section three (interaction with the truck). He paid attention to the robot 94.9 percent of the section length, as opposed to 79.8 percent when being exposed to the truck. Adam is the most able child in the group, and he has consistently expressed great interest in the robot, and robotics in general (he is a keen viewer of the TV show 'Robot Wars'). Given his interest and verbal abilities, he usually spends a substantial amount of time during the trials questioning the experimenters about the robot. Thus, when exposed to the robot he is tremendously interested in the robot, but he will not necessarily play with and look at the robot. He rather verbally interprets the robot's behaviour and inquiries about how it is programmed and how it works in general. Explicit eye gaze is therefore directed more away from the robot, although the video data shows that the robot is clearly a focus of attention. This example shows that a widely context-free statistical analysis does not necessarily yield 'meaningful' results. A combination of different criteria (not only eye gaze), and their interpretation in con-

\footnotetext{
${ }^{2}$ 2-tailed Mann-Whitney U Tests after finding no significant first-order autocorrelations (Kendall) among the duration of subsequent gaze bouts within conditions.

${ }^{3}$ Fisher Combination Test, $\chi^{2}=42.451, d f=12, p=3 \mathrm{E}-05$.
} 
text is vital. This is a reason why we are also applying Conversation Analysis (a very much context based qualitative technique) to the data [7], see discussion in section 4 .

Note, that the large standard deviation for Oscar (in the robot case) is explained by the fact that he looked at the robot only briefly a few times (within 7 minutes and 16 seconds overall section length) and then stared at the robot once for about 2.5 minutes and again for about 3 minutes. This is reflected in figure 3 which shows for Oscar substantially higher percentages (total eye gaze behaviour lengths divided by total section length) for the robot condition than for the truck condition. The data for Oscar in the truck condition in figure 2 is based on one measurement only: he looked at the truck once for 19 seconds, then the section was cut short due to an obvious lack of interest. This section with the truck lasted just 2 minutes 14 seconds in total. Statistical comparisons between truck/robot condition for eye gaze were therefore not possible for Oscar.

Interestingly, a xy-plot shown in figure 4 indicates some tendencies within group one (circles) with regard to the histogram shown in figure 2 , namely a potential positive correlation between the average behaviour lengths of 'robot' and 'truck' trials. One possible interpretation could be that this reflects (in group one) a general behavioural tendency that applies to the robot as well to the truck (and, as one might speculate) could apply to other objects as well. To put it simply, some children might in general show more eye gaze behaviour directed to objects than other children.

Eye gaze behaviour is only one of the criteria that we study. Particularly interesting could be a comparison with related criteria such as attention, see discussion of a concrete example above. The full analysis of the data is beyond the scope of this article and will be discussed in a forthcoming paper. Currently we also apply the technique to interactions of autistic children with a small humanoid robot in trials that are described in [5]. Other very interesting avenues for further analysis in the Aurora project are the identification of behaviour patterns, e.g. touch might or might not regularly be preceded by eye gaze and followed by handling. Also, a variety of other data analysis methods could be applied, cf. [10], [16].

\section{Conclusion}

This paper discussed a quantitative technique for analysing robot-human interactions. The application of the technique was demonstrated by presenting results on eye-gaze behaviour in a comparative study we did with autistic children. We found common ten- dencies among the seven children studied, but also clear differences that will impact future work in the project, e.g. when robots need to be tailored to the skills and needs of individual children. In order to characterise robot-human interactions a variety of criteria need to be considered, in particular crosscorrelations and temporal interdependencies among these. The list of criteria we suggested is based on relatively low-level, micro-behaviours, and are themselves not autism specific, e.g. could be applied to any other application area that involves robothuman interactions, e.g. service robotics. Moreover, the list of criteria can be adapted easily to the needs of particular application areas and research questions.

Our approach to evaluate robot-human interaction is inherently observational, and purely based on video data. Generally, observational data of robothuman interactions seems particularly useful in areas where direct inquiry (e.g. interviews, questionnaires) is not suitable (e.g. in certain therapeutic contexts as we discuss in this paper), where it is too intrusive (e.g. when young children are involved), or when responses are very likely to be biased by influences from the experimenters or attitudes or expectations of the subjects on the outcome of the study. Observational data can be analysed with quantitative and qualitative techniques. As we indicated above, there are limitations to any quantitative, statistical approach based on frequency and time duration measures. The particular context in which a behaviour occurs is often fundamentally crucial. For example, a child might show a particular behaviour only once, but this might have important therapeutic implications. Also, the specific spatial and in particular social context is important. For these reasons we also pursue a complimentary qualitative approach with Conversation Analysis (CA). Initial results are very promising and suggest that CA is particularly suited to reveal the interactional and communicative com petencies of autistic children [7].

The particular quantitative technique that we described in this paper is only part of a range of qualitative and quantitative techniques that we consider necessary for getting results that reflect the richness of the video data. Some of the techniques, such as $\mathrm{CA}$, require intensive specific training, other techniques, such as the quantitative technique discussed in this paper, require much less specific training. We also suggest that the same applies to other application areas where a precise analysis of the interactions is needed. We hope that the work presented in this paper will contribute to the advancement of research into humanoid or non-humanoid social robots that are intended to operate in human-inhabited environ- 
ments.

\section{Acknowledgments}

This project is supported by an EPSRC grant (GR/M62648). The robotic platform used is kindly donated by Applied AI Inc, and we are grateful for the continued support of the staff and pupils of Radlett Lodge School. Thanks to all members of the Aurora team for useful discussions. René te Boekhorst helped tremendously with the statistical analysis.

\section{References}

[1] AURORA. Url: http://www.aurora-project.com/. Last referenced on 11th of June, 2002, 2002.

[2] Cynthia Breazeal. Sociable machines: Expressive social exchange between humans and robots. $\mathrm{PhD}$ Thesis, MIT Department of Computer Science and Electrical Engineering, 2000.

[3] K. M. Colby and D. C. Smith. Computers in the treatment of nonspeaking autistic children. Current Psychiatric Therapies, 11:1-17, 1971.

[4] Kerstin Dautenhahn. Robots as social actors: Aurora and the case of autism. In Proc. CT99, The Third International Cognitive Technology Conference, August, San Francisco, pages 359-374, 1999.

[5] Kerstin Dautenhahn and Aude Billard. Games children with autism can play with robota, a humanoid robotic doll. In S. Keates, P. M. Langdon, P.J. Clarkson, and P. Robinson, editors, Universal Access and Assistive Technology, pages 179-190. Springer-Verlag London, 2002.

[6] Kerstin Dautenhahn and Iain Werry. Issues of robothuman interaction dynamics in the rehabilitation of children with autism. In J.-A. Meyer, A. Berthoz, D. Floreano, H. Roitblat, and S. W. Wilson, editors, Proc. From animals to animats 6 , The Sixth International Conference on the Simulation of Adaptive Behavior (SAB2000), pages 519-528, 2000.

[7] Kerstin Dautenhahn, Iain Werry, John Rae, Paul Dickerson, Penny Stribling, and Bernard Ogden. Robotic playmates - analysing interactive competencies of children with autism playing with a mobile robot. In K. Dautenhahn, A. Bond, L. Cañamero, and B. Edmonds, editors, Socially Intelligent Agents - Creating Relationships with Computers and Robots, pages 117-124. Kluwer Academic Publishers, 2002.

[8] Allison Druin and James Hendler, editors. Robots for Kids - Exploring new technologies for learning. Morgan Kaufmann Publishers, 2000.

[9] Rita Jordan. Autistic Spectrum Disorders - An Introductory Handbook for Practitioners. David Fulton Publishers, London, 1999.

[10] Philip N. Lehner. Handbook of ethological methods. Garland STPM Press, New York, 1979.
[11] F. Michaud, A. Clavet, G. Lachiver, and M. Lucas. Designing toy robots to help autistic children - an open design project for electrical and computer engineering education. Proc. American Society for Engineering Education, 2000.

[12] D. Moore. Computers and people with autism. Communication, pages 20-21, 1998.

[13] Seymour Papert. Mindstorms: Children, Computers, and Powerful Ideas. Basic Books, New York, 1980.

[14] C. Plaisant, A. Druin, C. Lathan, K. Dakhane, K. Edwards, J. M. Vice, and J. Montemayor. A storytelling robot for pediatric rehabilitation. Proc. ASSETS '00, Washington, Nov. 2000, ACM, New York, pp. 50-55, 2000.

[15] S. Powell. The use of computers in teaching people with autism. In: Autism on the agenda: papers from a National Autistic Society Conference, London, 1996.

[16] Colin Robson. Real world research: A resource for socal scientists and practitioner- researchers. Blackwell, Oxford, 1993.

[17] C. Tardiff, M.-H. Plumet, J. Beaudichon, D. Waller, M. Bouvard, and M. Leboyer. Micro-analysis of social interactions between autistic children and normal adults in semi-structured play situations. International Journal of Behavioural Development, 18(4):727-747, 1995.

[18] S. Weir and R. Emanuel. Using LOGO to catalyse communication in an autistic child. Technical report, DAI Research Report No. 15, University of Edinburgh, 1976.

[19] Iain Werry, Kerstin Dautenhahn, and William Harwin. Investigating a robot as a therapy partner for children with autism. Proc. AAATE 2001, 6th European Conference for the Advancement of Assistive Technology (AAATE 2001), 3-6 September 2001 in Ljubljana / Slovenia., 2001.

[20] Iain Werry, Kerstin Dautenhahn, Bernard Ogden, and William Harwin. Can social interaction skills be taught by a social agent? the role of a robotic mediator in autism therapy. In M. Beynon, C. L. Nehaniv, and K. Dautenhahn, editors, Proc. CT2001, The Fourth International Conference on Cognitive Technology: Instruments of Mind, LNAI 2117, pages 57-74, Berlin Heidelberg, 2001. Springer-Verlag. 